\title{
DIABETES \\ Blocking RANKL signalling might prevent T2DM
}

Blockade of receptor activator of nuclear factor $\kappa \mathrm{B}$ ligand (RANKL) signalling in hepatocytes protects against type 2 diabetes mellitus (T2DM), report researchers.

"It is well known that activation of nuclear factor $\kappa \mathrm{B}(\mathrm{NF}-\kappa \mathrm{B})$ in the liver is a crucial event in the development of hepatic insulin resistance and T2DM," explains lead author Stefan Kiechl of the Medical University of Innsbruck, Austria. "RANKL, a member of the tumour necrosis factor superfamily, is a potent activator of NF- $\kappa \mathrm{B}$, and its receptor RANK is expressed on liver cells. We, thus, hypothesized that RANKL is involved in hepatic NF- $\kappa B$ activation, leading to T2DM."

The researchers studied the association between serum levels of soluble RANKL and osteoprotegerin and subsequent risk of developing T2DM in 844 men and women without T2DM aged 40-79 years. Soluble RANKL was assessed because it has been shown to be functionally active.

During follow-up, between 1990 and 2005, 78 individuals of the cohort developed T2DM. Baseline levels of soluble RANKL between individuals who had and had not developed T2DM differed considerably: risk of T2DM was elevated in the group with the top tertile of concentrations of soluble RANKL compared with the group with the bottom tertile (OR 4.06, 95\% CI 2.01-8.20). Adjustment for lifestyle factors and body composition did not significantly affect the risk association. Interestingly, although concentrations of osteoprotegerin were not elevated preceding T2DM onset, as they were for soluble RANKL, increased levels were found in individuals after disease occurrence.

In a series of mouse models in which RANKL signalling was downregulated systemically or in the liver, the investigators showed that hepatic insulin sensitivity and plasma glucose concentrations improved with blockade of RANKL signalling. In one such experiment, mice with a hepatocytespecific Rank knockout were fed a high-fat diet for 4 weeks. These mice did not develop insulin resistance, whereas control mice did.

The investigators note that medications for T2DM already available, such as metformin, lower RANKL activity in bone and might also lower RANKL activity in the liver. They speculate that RANKL antagonism could be a yet unknown antidiabetic mechanism of metformin.

For Kiechl and co-workers, the next important step is the development

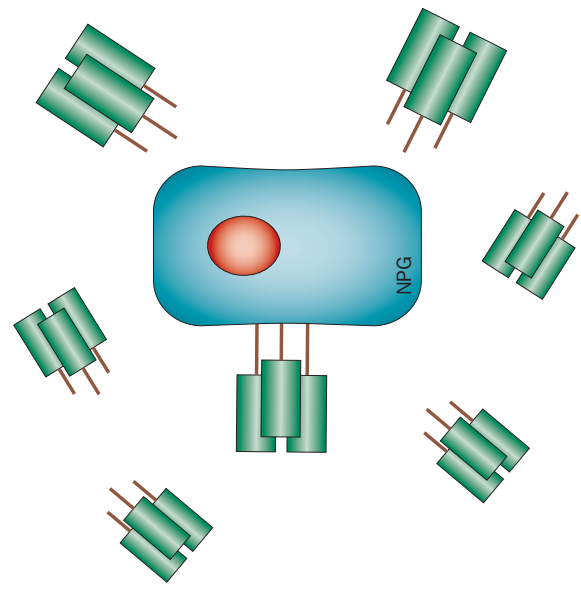

and testing of specific anti-RANKL medications for the treatment and prevention of T2DM in humans. "Routine application of anti-RANKL strategies in current osteoporosis therapy (available for years) may help to speed up this process," says Kiechl.

Carol Wilson

Original article Kiechl, S. et al. Blockade of receptor
activator of nuclear factor- $\mathrm{KB}$ (RANKL) signaling improves
hepatic insulin resistance and prevents development of
diabetes mellitus. Nat. Med. doi:10.1038/nm.3084

